Bioética

\section{ÉTICA NA PESQUISA COM SERES HUMANOS}

Recentemente, a Editora Loyola, juntamente com o Centro Universitário São Camilo e a Sociedade Brasileira de Bioética, publicaram o livro: "Bioética: Poder e Injustiça", preciosa coletânea das mais importantes conferências apresentadas no VI Congresso Mundial de Bioética, realizado em Brasília em 2002.

São 45 textos de autoridades de renome internacional da área de bioética, representando autores de 24 países dos cinco continentes. Queremos destacar o artigo de Corina Bontempo que apresenta a experiência brasileira sobre ética na pesquisa com seres humanos. Ressalta a autora que a missão da Comissão Nacional de Ética em Pesquisa (CONEP) está em garantir e resguardar a integridade, os direitos e liberdades fundamentais dos voluntários participantes de pesquisas desenvolvidas no território nacional.

\section{Comentário}

Temos no Brasil, atualmente, mais de 400 comitês institucionais de ética em pesquisa (CEP), o que significa, minimamente, 4.000 pessoas diretamente envolvidas no processo de avaliar projetos de pesquisa realizados no país.

A Gazeta Mercantil, de junho de 2000, apresentou dados da indústria farmacêutica registrando expressivo aumento de investimentos estrangeiros em pesquisas no Brasil. Foi estimado destinação de 78 milhões de dólares para investigação de novos medicamentos no mencionado ano.

Publicação veiculada pelo jornal Washington Post, aponta para uma maior procura por parte de grandes empresas multinacionais farmacêuticas para encontrar parceiros em países periféricos para realização de pesquisas com novas drogas, mencionando-se especificamente Brasil, México, Argentina, países do leste europeu e África do Sul.

Estamos, portanto, na condição de território de pesquisadores com adequada formação científica e centro eficiente de recrutamento de pessoas para execução de qualquer projeto de pesquisa, o que é mister salientar, não tem ocorrido nos países centrais.

A experiência brasileira nesse campo tem sido exemplar, pois conseguimos estruturar um sólido sistema de proteção aos sujeitos de pesquisa que está fundado na Resolução 196/96 do Conselho Nacional de Saúde.

Imperioso, porém, é considerar que muitos são os desafios ainda persistentes, quais sejam: independência dos CEPs diante de interesses de pesquisadores, instituições e patrocinadores, maior legitimidade dos CEPs, adequado controle social das pesquisas e, apenas para ficar em alguns poucos exemplos, maior representação de usuários nos CEPs.

\section{José Eduardo de Siqueira}

Referência

Freitas CBD. Ética na pesquisa com seres humanos:a experiência brasileira. In: Garrafa V, Pessini L. Bioética: poder e injustiça. São Paulo: Loyola; 2003.

\section{Medicina Baseada em Euidências}

CONTROLE DA GLICEMIA NA

FASE AGUDA DO ACIDENTE
VASCULAR CEREBRAL

Há relativo consenso de que hiperglicemia (glicemia > $120 \mathrm{mg} / \mathrm{dL}$ ) é deletéria na fase aguda do acidente vascular cerebral (AVC), independentemente da idade do paciente ou da extensão e tipo do AVC (isquêmico ou hemorrágico) 1,2(B) 3,4(D). O diabetes pode piorar o prognóstico do AVC por favorecer o desenvolvimento de complicações clínicas no curso da doença. Não há evidência direta de que o controle estrito da glicemia com insulinoterapia nos primeiros dias do infarto cerebral altere a evolução clínica a curto ou longo prazo, mas esta evidência já é clara em estudos de infarto agudo do miocárdio (estudo DIGAMI) ${ }^{5}(A)$. Recomenda-se, então:

- Pesquisar diabetes em todo paciente em que se detecte hiperglicemia; na ausência de diagnóstico prévio, medir hemoglobina glicosilada (total e fração AI);
- Monitorizar a glicemia nas primeiras 48 a 72 horas em todo paciente com AVC agudo (qualquer tipo); sugere-se intervalo de 6 horas;

- Regimes de monitorização mais intensivos e por tempo maior podem estar indicados em pacientes com hiperglicemia grave ou história de diabetes tipo I - insulino dependente, hiperglicemia de difícil controle ou risco de hipoglicemia; - Evitar soluções parenterais de glicose;

- Soluções cristalóides (soro fisiológico a 0,9\% com potássio ou Ringer) ${ }^{5}(A)^{4}(B)$ é sugerido para reposição volêmica parenteral;

- Suporte nutricional na fase aguda de preferência por via digestória no menor prazo possível;

- Evitar e tratar causas adicionais de hiper glicemia, como o uso de esteróides ou infecção;

- Evitar hipoglicemia e outras alterações metabólicas; dar glicose hipertônica em pacientes com glicemia menor que 70 mg\%; atenção especial em pacientes em uso de antidiabéticos orais;

Até que mais dados imponham outras recomendações, o uso rotineiro de insulina por infusão contínua não pode até aqui ser recomendado; sugere-se, entretanto, o uso de doses isoladas de insulina regular sempre que os níveis glicêmicos excedam 180 mg\%; em ambientes sofisticados que permitam monitorização intensiva, níveis mais baixos, como 150 mg\%, podem ser utilizados.

\section{Comentário}

Ao lado do tratamento específico, os cuidados gerais, e aqui se inclui o controle da glicemia, são de fundamental importância no tratamento do doente com AVC. São medidas em geral simples, baratas, fácies, imediatas e que trazem bons resultados. $\mathrm{O}$ doente com AVC com alguma freqüência tende a evoluir com hiperglicemia, o que é comprovadamente prejudicial, pois aumenta a área de isquemia. Estes doentes, muitas vezes pelo estresse da doença ou por infecções, descompensam ou agravam um diabetes prévio ou então recebem medicação glicosada ou que favoreça a hiperglicemia. Estas situações devem ser cuidadosamente analisadas e afastadas, procurando-se manter os níveis glicêmicos dentro de valores normais. É um procedimento que pode ser conseguido em qualquer local, mesmo que não disponha de sofisticados recursos, deve ser efetivado imediatamente, e isoladamente contribui para o prognóstico do doente.

Rubens José Gagliardi 
Referências

I.Toni D, De Michele M, Fiorelli M, Bastianello S, Camerlingo M, Sacchetti ML, et al. Influence of hyperglycemia on infarct size and clinical outcome of acute ischemic stroke patients with intracranial arterial occlusion. J Neurol Sci 1994; 123: 129-33. 2. Li PA, Shuaib A, Miyashita H, He QP, Siesjo BK, Warner DS. Hyperglycemia enhances extracellular glutamate accumulation in rats subjected to forebrain ischemia. Stroke 2000; 31: 183-92. 3. Caplan LR. Introduction and perspectives. In: Caplan LR, editor. Caplan's stroke: a clinical approach. Boston: Butterworth-Heinemann; 2000. p.3-16.

4. Caplan LR. Clinical diagnosis and general laboratory evaluation. In: Welch KMA, Caplan LR, Reis DJ, Siesjö BK, Weir B, editors. Primer on cerebrovascular diseases. San Diego: Academic Press 1997. p.593-7.

5. Malmberg K, Norhammar A, Wedel H, Ryden L. Glycometabolic state at admission: important risk marker of mortality in conventionally treated patients with diabetes mellitus and acute myocardial infarction: long-term results from the Diabetes and Insulin-Glucose Infusion in Acute Myocardial Infarction (DIGAMI) study. Circulation 1999; 99:2626-32.

\section{Graus de Recomendações}

(A) Estudos experamentais ou observacionais de maior consistência

(B) Estudos experimentais ou observacionais de menor consistência

(C) Relato de casos (estudos não controlados)

(D) Opinião desprovida de avaliação crítica, baseada em consertos, estudos fisiológicos ou modelos animais.

\section{Medicina Baseada em Euidências}

\section{O MANEJO DAS SÍNDROMES DOLOROSAS MIOFASCIAIS}

Dor é o motivo mais comum para os pacientes procurarem assistência médica, e a síndrome dolorosa miofascial é a causa imediata ou o componente mais freqüente da dor músculo-esquelética '. O manejo dos pacientes com síndromes dolorosas miofasciais é um dos problemas mais importantes encontrados na prática clínica.

O quadro de dor referida em uma região do corpo relacionada à presença de pontos-gatilho ativos, comum nos serviços de emergência, tende a se tornar crônico. Afeta pacientes que se apresentam com queixas como cefaléia, torcicolo e lombalgia (Figuras I, 2 e 3).

\section{Figura I - Mecanismos da síndrome dolorosa miofascial. Adaptado de Melzack R., Wall P.D., Textbook of Pain. 3 ed, Churchill Livingstone, UK, 1994}
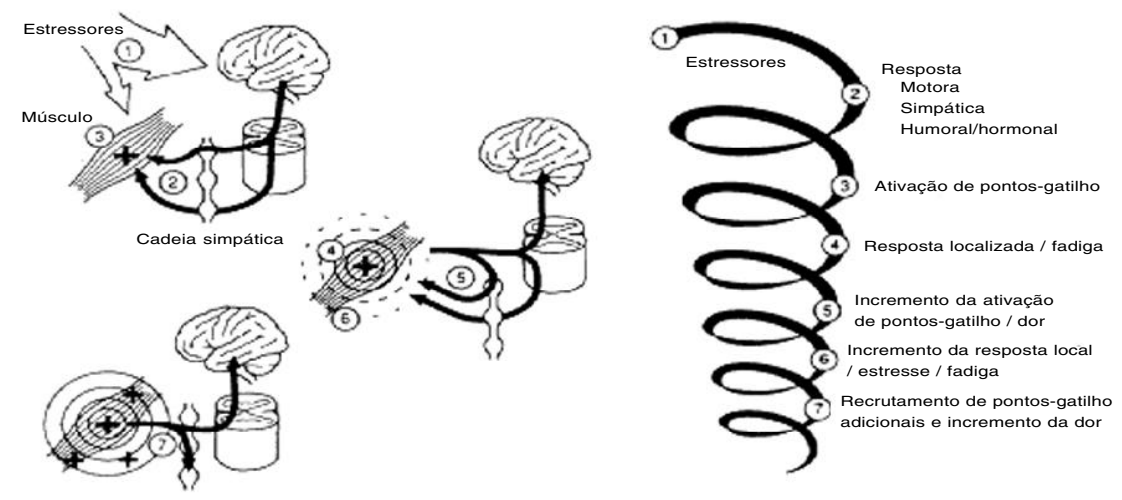

Figura 2 - Exemplos de sentidos de propagação da dor referida a partir de ponto-gatilho. Adaptado de Travell \& Simons, Myofascial Pain and Dysfunction - The Trigger Point Manual. I ed., William and Wilkins, USA, 1983
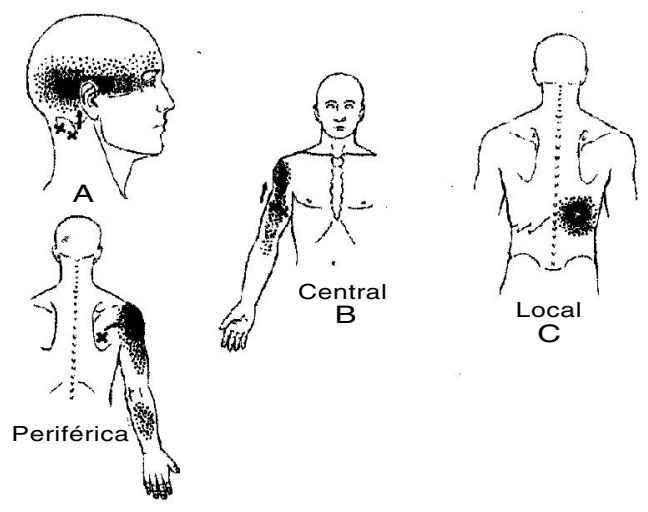

\section{Figura 3 - Padróes de dor referida de músculos mais comumente encontrados em pacientes com cefaléia. Adaptado de Steven B., Graff-Radford, D.D.S. Regional Myofascial Pain Syndrome and Headache: Principles of Diagnosis and Management. Current Pain and Headache Reports 5:376-38I, 2001.}

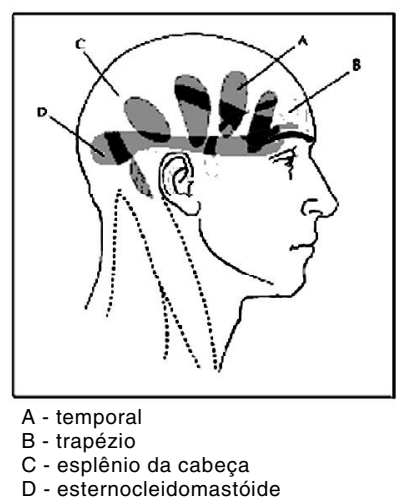

